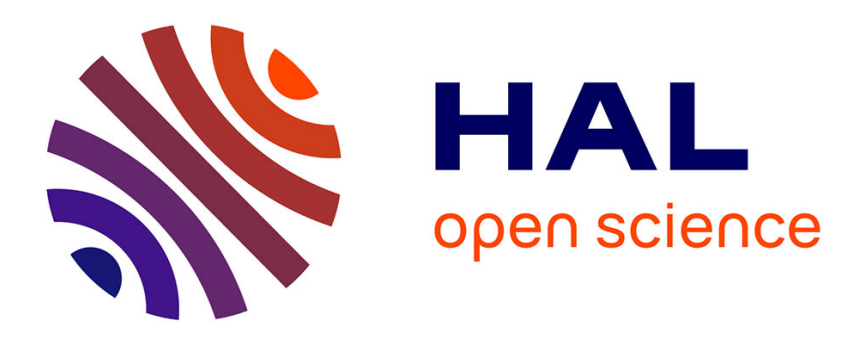

\title{
Mains levées, mains supines, à propos d'une base funéraire de Kadyanda (Lycie)
}

\author{
Marie-José Morant
}

\section{To cite this version:}

Marie-José Morant. Mains levées, mains supines, à propos d'une base funéraire de Kadyanda (Lycie). KTĖMA Civilisations de l'Orient, de la Grèce et de Rome antiques, 1999, 24, p. 289-294, 2 pl. halshs-00001459

\section{HAL Id: halshs-00001459 \\ https://shs.hal.science/halshs-00001459}

Submitted on 15 Apr 2004

HAL is a multi-disciplinary open access archive for the deposit and dissemination of scientific research documents, whether they are published or not. The documents may come from teaching and research institutions in France or abroad, or from public or private research centers.
L'archive ouverte pluridisciplinaire HAL, est destinée au dépôt et à la diffusion de documents scientifiques de niveau recherche, publiés ou non, émanant des établissements d'enseignement et de recherche français ou étrangers, des laboratoires publics ou privés. 


\section{Mains levées, mains supines, à propos d'une base funéraire de Kadyanda (Lycie)}

Rrsumr. Une base funéraire inscrite retrouvée à Kadlanda (Lycie) évoque le souvenir de Rhodopè, sans doute figurée sur le monument et entourée par deux mains levées. La présence de ces mains pose diverses questions : à qui appartiennent-elles? Dans quel but sont-elles représentées? L'expression «mains supines» (manus supinatae) employée pour les décrire dans la plupart des publications françaises peutelle s’appliquer aussi systématiquement que cela a été fait?

Zusamminfassung. - Eine kleine Grabsäule, die in Kadyanda (Lykien) gefunden wurde, erwähnt die verstorbene Rhodope, die wahrscheinlich auf dem kleinen Denkmal mit zwei erhobenen Händen dargestellt wird. Wem gehören diese Hände? Welche Rolle spielen sie? Sind sie supinatae manus, Fluchhände? Eine klare Definition muß3 noch hergestellt werden.

Lors d'une des campagnes de prospection en Lycie dirigées par Ed. Frézouls a été découverte à Kadvanda, cité lycienne au nord-est de Telmessos, à proximité immédiate de la grande nécropole méridionale, une base cylindrique funéraire en calcaire ( ${ }^{1}$ ). Elle a été décrite et son inscription a été publiée dans un article touchant d'autres inédits de la même ville $\left(^{2}\right)$. Mais comme le commentaire en fut nécessairement succinct, il ne m'a pas semblé inutile de reprendre ce dossier, d'autant que ce site est, je crois, un de ceux qu'aimait notre chef de mission. Les efforts physiques qu'il devait fournir pour y accéder à chacun de nos séjours, mais aussi les brefs repos qu'il y prenait après nos déjeuners frugaux, allongé à même la pierre en bordure du «stade», à l'ombre des pins, dans un silence quasi religieux, ne devaient pas y être étrangers.

\section{DFSCRIPTION DE 1.A BASE FUNFRAIRE DF. KADYANDA (fig. 1)}

\section{- L'inscription}

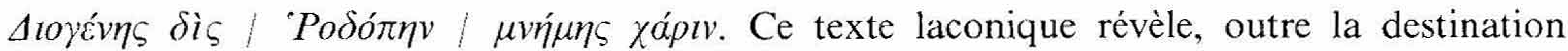
funéraire du petit monument, deux noms. On ne reprendra pas l'étude onomastique de Diogénès, très fréquent en Lycie. Mais le nom de la défunte, Rhodopè est beaucoup plus rare, du moins dans cette province comme nous l'avions déjà souligné. Nom d'une Océanide, compagne de Corè, il évoque aussi celui d'une chaîne de montagne au sud-ouest de la Thrace, région devenue province au Bas-Empire et notée par J. Annequin comme terre de magie $\left({ }^{3}\right)$. I.es dimensions

(1) Cet autel a été découvert par Sylvie Dardaine, membre de la mission, que je remercie vivement pour ses conseils prodigués au cours de cette recherche. Je remercie également Anne Jacquemin pour sa relecture utilement critique.

(2) Vingt-huit au total. Ed. Fríot1.s, M.-J. Morani, «Inscriptions de Sidyma et Kadyanda (II)», Ktèma, 11 , 1986 [1990]. pp. 239-253. La base dont il va être question ici correspond au n ${ }^{\circ} 22$ de l'article de Ktèma, 11.

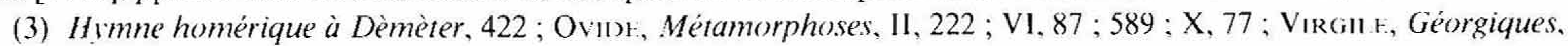
III, 351; IV, 461. Cf. J. ANNIQIIN, Recherches sur l'action magique et ses représentations (Ir - II' siècle ap. J.C.). Paris. 1973, $A L U B, 146$, p. 171. 
de cette base funéraire - 62 centimètres de hauteur totale et un diamètre maximum de 35 centimètres - l'absence de généalogie, mis à part $\delta i$, et de nomon excluent d'emblée que Rhodopè ait appartenu à l'aristocratie locale. Parmi les trois autres Rhodopè mentionnées en Lycie celle dOOlmpos (TA.M, 1133) est une esclave, mais les deux de Temmesses sont de milieu beaucoup plus aisé. L'une, épouse d'un citoyen romain, Klaudios Kirpos, a une famille connue par ailleurs (TAM, 234) $\left({ }^{4}\right)$; lautre offre sur un sarcophage une inscription élégiaque à son chien, révélant, outre des moyens financiers certains, un goüt marqué pour la poésie $\left({ }^{5}\right)$. Une recherche plus systématique et plus approfondie - essenticllement pour dater chaque inscription - - permettrait peut-être de déceler l'évolution d'une mode dans l'emploi régional de ce nom (6).

L'absence de liens de parenté entre Diogénès et Rhodopè ne signifie pas nécessairement que la défunte était une célibataire ou une concubine sans enfant : la situation familiale n'est pas toujours mentionnée sur les épitaphes (7) et une étude récente sur des villes d'Asie Mineure souligne l'importance des liens, en dehors de ceux de la famille nucléaire, apparaissant dans les inscriptions $\left({ }^{8}\right)$.

En l'absence de nomina significatifs seule la forme des lettres permet, par comparaison avec d'autres inscriptions datées du site ou des environs, d'envisager une datation que nous situerions entre la deuxième moitié du ${ }^{\mathrm{E}}$ et le $\|^{\mathrm{E}}$ siècle ap. I.-C. Si le sigma est déjà lunaire l'epsilon ne l'est pas encore et la petite barre transversale de l"alpha est encore brisece.

\section{- Lepersonnage}

Il semblait logique que le petit personnage sculpté sur la base fût une représentation de la défunte. On notera l'absence de niche ou de tout cadre centré sur le personnage. Un bandeau circulaire en creux d'où se détache le relief du corps n’inclut pas la tête. Sur un peplos dont on aperçoit les plis verticaux au-dessus des pieds, pieds dont on ne saurait dire s'ils sont chaussés, cette femme porte un himation dont on reconnaît les plis en diagonal. Les bras et les épaules semblent pris par une sorte d'étole, peut-être une calyphe. À l'hypothèse d'une longue chevelure, proposće lors de la précédente publication nous préférons voir la tête couverte par un voile ou par un pan de l'himation: le bas du voile s'enfonçant sous la calyphe $\left({ }^{9}\right)$. De façon très

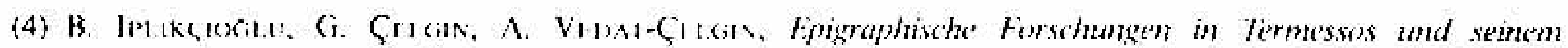
Ferriforium. [1], Vienne, 1994, Osterreichische Akademie der Wissenschalten. philosophisch- historische Klasse, Sitzungsberichte, $610, \pi^{\prime \prime} 19$.

(5) B. Im IKclocil U, "Epigraphische Forschungen im antiken Termessos und seinem Territurium". Hundert Jahre" kleinasiatische Kommission der asterreichischen Akademie der Wissenschaften. Akten des Stmposiums von 23. his

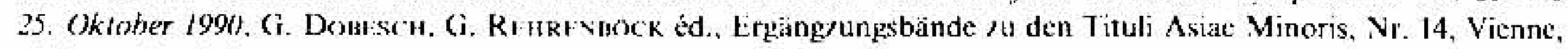
1993. pp. 26(1)-262.

(6) Pour l'étude de ce nom dans les provinces hellénophones de llimpirc: A Texikon of Greek Personal Names. I. (The degcan Isiands. Cypras. Cirenaica), Oxford. 1987: une occurrence en (yrinaïque: III (The Pelopomnexe, Western Greete, Sicily and Magna Graecia). Oxford. 1997. cing occurrences (Sicile. Italie du Sud. Epire). F. Pkilisicikı. Nomenhuth. Amsterdam, 1967, recense pour l'Égypte deux fois le nom Rhodopi dans des papyrus. Ce nom niest pas incomnu dans les provinces de langue latine, on le retrouve par exemple in Fspagne (At, 1994. 818 et 1075). Pour l'étude de ce nom a Rome voir H. Soll, Die griechischen Personentamen in Rom. Ein Namenhuch, 1, Burlin -

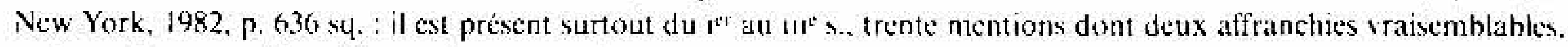
quatre csclaves ou affranchies et vingt-quatre incernae.

(7) Iin Lycic, on trouve dautres funćraires sans precision de parente, par ex. TAM, 383, 597,627, 807 ou 1076.

(8) D), B. MARTIX, «The Construction of the Ancient Family: Methodological Considerationsm, IRS. 86. 1996. pp. $40-60$.

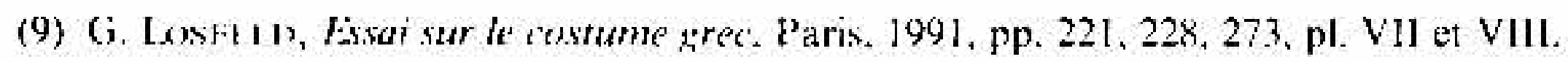


courante les bras sont pliés : la main droite posée à gauche, sur le haut de la poitrine, et la main gauche paraissant cachée par le coude et l'avant-bras droit. Dans le monde grec le port du voile évoque une femme mariée, mais la position debout, l'absence de tout compagnon (conjoint, servante, enfant) plutôt une jeune fille $\left({ }^{10}\right)$. Le visage a été abîmé, peut-être même martelé, et la photographie laisse soupçonner la trace d'une coiffe, sorte de calathos, dont le martelage aurait été encore plus profond que celui opéré sur le visage. Serait-on en présence d'une divinité dont les pieds reposent aussi sur un socle ? Cela est peu probable ("I). Déjà à l'époque classique le calathos caractérisait sur les stèles les activités d'une maîtresse de maison mais pouvait aussi être utilisé pour désigner la tombe d'une jeune fille $\left({ }^{12}\right)$.

\section{- Les deux mains}

De part et d'autre du petit personnage et très proche de lui se dressent deux grandes mains avec chacune une longue partie d'avant-bras. Celle qui est située à droite, à la différence de son pendant de gauche très abîmé, est relativement bien conservée $\left({ }^{13}\right)$. Il s'agit de mains assez fortes, paumes face au spectateur comme elles le sont souvent du moins quand la représentation est assez réaliste pour qu'on puisse le déceler. Mais à la différence de la majorité des mains levées la main placée à droite a le pouce à l'extérieur, comme on peut le supposer aussi pour celle de gauche. Sur la trentaine de stèles et sarcophages ornés de mains du monde grec oriental et essentiellement recensés par E. Pfuhl et $\mathrm{H}$. Möbius seules deux stèles - peut-être trois - offrent la même particularité : une d'origine précise inconnue mais très certainement de l'Orient grec $\left({ }^{14}\right)$ (fig. 2), la seconde d'Enkomi (Chypre) avec là aussi une importante partie d'avant-bras $\left({ }^{15}\right)$ (fig. 3); la conservation de la troisième, retrouvée à Sofular, site de Kibyratide assez proche de Kadyanda, est trop mauvaise pour que l'on soit totalement sûr de l'emplacement des pouces $\left({ }^{16}\right)$. À qui sont ces mains? Appartiennent-elles au mort ? Dans ce cas si les paumes sont présentées les pouces sont à l'intérieur et sur certaines stèles le défunt ou la défunte sont représentés les bras levés $\left({ }^{17}\right)$ (fig. 4). Les reliefs avec paumes et pouces à l'extérieur sont-ils simplement une maladresse de sculpteur?

(10) G. HoffMANN, "La jeune fille et la mort : quelques stèles à épigramme», Annali Istituto Universitario Orientale Napoli, Sez. Archeologia e Storia Antica (= AION (archeol)), Sezione tematica: la parola, l'immagine, la tomba, Atti del colloquio internazionale di Capri, X, 1988, p. 81.

(11) Découverte à Oinoanda d'une stèle votive avec représentation féminine fort proche dans l'habillement de celle de notre base. Elle est interprétée comme la déesse pisidique dont une des fonctions était la garde des tombeaux. L. Roвert, Villes d'Asie Mineure. Etude de géographie antique, Paris, 1935, p. 212 ; N. P. Milner, M. F. Sмmith, "New Votive Reliefs from Oinoanda", Anatolian Studies, 44, 1994, pp. 65-76. On remarquera seulement que les auteurs de l'article proposent de dater les inscriptions - plusieurs sur la stèle - des $\|^{e}-1 I^{e} \mathrm{~S}$. ap. J.-C. Si la représentation féminine est contemporaine des dédicaces, elle le serait aussi de notre base de Kadyanda. La mode vestimentaire a pu être la même pour les représentations de mortelles ou d'immortelles. Reste le problème des attributs tenus par la déesse à Oinoanda et qui n’apparaissent pas à Kadyanda. Cet argument parât déterminant pour abandonner l'idée d'une représentation divine sur notre base, non celle d'une défunte héroïsée.

(12) M.-Th. Le Dinahet-Counloud, N. Mouret, «Les stèles funéraires grecques. Études stylistiques et iconographiques. Années 1980-1992", Topoi, 3, 1993, p. 118.

(13) Comme les représentations de paires de mains levées figurent toujours une main gauche et une main droite identiques - à l'exception du monde punique, voir infra addendum - nous partirons de ce principe pour restituer la main abîmée comme l'exact pendant de celle qui est conservée.

(14) E. Pғинц, Н. Möвıs, Die ostgriechischen Grabreliefs, Mayence, 1977, $\mathrm{n}^{\circ} 433$.

(15) F. Cumont, «Deux monuments des cultes solaires», Syria, XIV, 1933, p. 388.

(16) Chr. NAour, "Inscriptions et reliefs de Kibyratide», ZPE, 22, 1976, p. 122, $\mathrm{n}^{\circ} 14$.

(17) E. Pfuhl, H. Möbius, op. cit., nos 1019 et 1020 et R. P. Mouterde, «Inscriptions grecques conservées à l'Institut français de Damas», Syria, Vl, 1925, p. 243 sq., n 32 . 
A notre connaissance toutes ces mains, quels que soient l'emplacement du pouce ou la face représentée, sont appelées "supines" dans les publications françaises $\left({ }^{18}\right)$. Cet adjectif est à rapprocher du latin supinare qui dans des textes antiques pouvait être adjoint à manus (19) pour décrire une position bien particulière: les bras sont levés, les mains tendues mais avec une notion de renversement, de retournement. Dans le vocabulaire physiologique le mot français "supination" est "un mouvement de rotation que la main et l'avant-bras exécutent de dedans en dehors, sous l'action des muscles supinateurs ; position de la main après ce mouvement" $\left({ }^{20}\right)$. $\mathrm{Si}$ on lève ses mains en position de supination elles se retrouvent paumes tournées vers soi, pouces à l'extérieur, exactement comme celles représentées sur la base de Kadyanda qui seraient alors celles du dédicant, du passant, de toute personne placée face à la sépulture.

\section{SIGNIHICATIONS DIS MAINS I.EVEFS}

Le catalogue déjá cité de Pfuhl et Möbius, surtout centré sur le monde grec oriental mais touchant l'ensemble des monuments funéraires, permet de recenser une trentaine de représentations de mains levées $\left(^{21}\right)$. I.a langue allemande les désigne par une expression unique, quelle que soit la position des pouces: «Fluchhände», que l'on peut traduire par mains de malédiction, d’imprécation. Une vingtaine, datées, seechelonnent entre lépoque hellénistique et le IV s. ap. J.-C. Les épitaphes qui les accompagnent sont courtes pour la plupart. lous les monuments sont funćraires : les formes sont diverses : stèles en majorité. sarcophages, autels quadrangulaires, bases cylindriques, tombes rupestres, mais on ne peut être sûr d'un lien initial entre ces divers éléments: forme de la sépulture. décor, inscription. les remplois étant très fréquents. L'emplacement des mains est lui aussi varié. En plus des mains levées de personnages dont nous avons parlé ${ }^{22}$ ) on trouve des mains udétachées", avec ou sans avant-bras, rassemblées en haut d'une stèle $\left({ }^{23}\right)$, de côté $\left({ }^{24}\right)$, sur le bas $\left({ }^{25}\right)$, séparées de part et d'autre d'une scène $\left({ }^{26}\right)$, d'un personnage $\left({ }^{27}\right)$. Enfin elles peuvent être le seul ornement d'un monument funéraire $\left({ }^{28}\right)$, accompagner un personnage seul, debout $\left({ }^{29}\right)$. couché $\left({ }^{30}\right)$ ou plusieurs personnages (père, mère, enfant) ( $\left.{ }^{3 t}\right)$, des scènes funéraires traditionnelles comme le couronnement d'une épouse par son mari $\left({ }^{32}\right)$, enfin une curieuse scène de labour $\left({ }^{33}\right)$.

(18) Voir par exemple J. et L. Roner1. Index du Bulletin epigraphique, III, Les mots français, Paris. 1975, s. u. amains supinesm. renvoyant mème à deux mains entrecroisées. Bull. ép., 1959, 273.

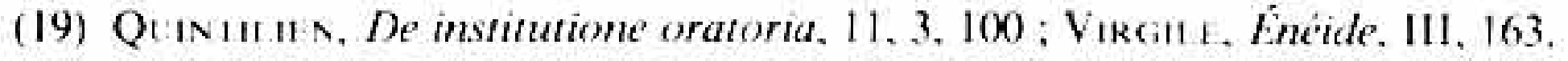

(20) Dictionnaire alphabétique et analegique de la langue française. I.. Rови R T. IV. Paris. 1978. s. us "supination".

(21) Nous y ajoutons deux découvertes plus récentes, celle qui a déjà été mentionné stupra note 16: Chr. Naot k. loc, cit.. p. 122, $\mathrm{n}^{\circ} 14 \mathrm{et} \mathrm{M}$. Ric1, "Inscriptions votives au Musée d'Eskisethir,. Jiva Antika, 44, 1994, p. 170 s4.. $\mathrm{n}^{\circ} 26$.

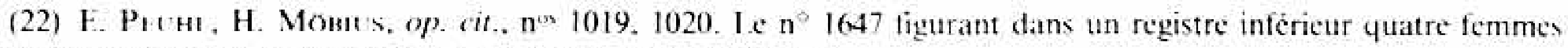
les mains levées n’a pas été retenu dans l'index $s, u$. «fluchhände"s.

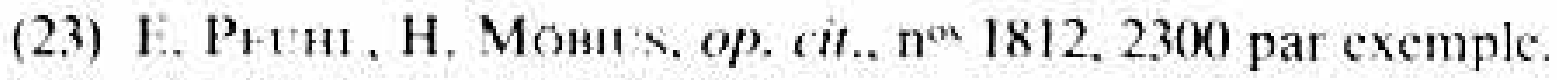

(24) Ins. ihid. op. cit, $\mathrm{n}^{\circ} 1675$.

(25) Chr. Nsouk, loc cit.

(26) L. Pнна, H. Mö! х, op. cit., n 1750.

(27) Iit., ibid., $\mathrm{n}^{\circ} 433$ par exemple.

(28) Li1,.. ithid., n"s 2246, 2248 .

(29) L11,.. ihid., n 433 .

(30) I11., ibid., n 1491.

(31) I11.. ibid. n 1812 .

(32) I11). ihid., $\mathrm{n}^{\prime \prime} 1675,1750$.

(33) In,. ihid.. n 1146. Fvocation du labour mythique dLebetlaeus? Voir it ee sujet M. H. JAMIson, "The Hero Echetlacusn. Transactions and Proceedings of the American Philolegical Associatsm. 82, 1951. pp. 49-61. 
Si les différentes recensions de représentations de mains levées à travers le monde romain faites par Cumont sont largement dépassées - les découvertes se sont multipliées depuis ses publications - sa réflexion demeure fondamentale $\left({ }^{34}\right)$. Geste d'imploration, de supplication, on tend les mains ouvertes vers le ciel. C'est le geste le plus habituel dans la prière antique ${ }^{35}$ ). Il donne un pouvoir magique, coercitif aux paroles prononcées $\left({ }^{36}\right)$. Divers textes littéraires le mentionnent (Tite Live, XXXVI, 9, 8; Virgile, Enéide, III, 173; IV, 203, etc.) et nombre

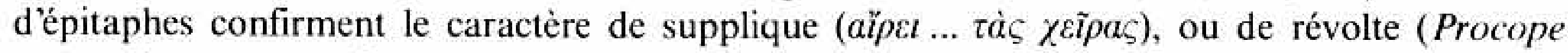
manus leuo contra deum qui me innocentem sustulit) $\left({ }^{37}\right)$ à la suite d'une mort violente, ou précoce $\left.{ }^{38}\right)$. Parmi les divinités invoquées Hélios revient fréquemment $\left({ }^{39}\right)$ et des précisions peuvent être données sur les circonstances de la mort (assassinat) $\left.{ }^{40}\right)$. On notera que tous ces éléments sont absents de l'épitaphe de Rhodopè.

Mais Cumont avait décelé une autre signification à la représentation des mains levées. Elles pourraient être celles du dieu qui protège $\left({ }^{41}\right)$. La petite base de Kadyanda pouvait facilement être dérobée, pour un remploi, par exemple. Elle pouvait aussi - et un grand nombre d'inscriptions nous le font savoir - être abîmée, simples gestes de vandalisme qui semblaient très fréquents. Or le monument funéraire est en quelque sorte l'image du corps du défunt : il est lavé, oint d'huile ${ }^{\left({ }^{42}\right)}$. En Anatolie et en Lycie surtout, où apparaissent les plus anciennes imprécations en langue grecque (au Ive s. av. J.-C. à Telmessos et à Antiphellos), l'idée de la mort et de la vie dans l'au-delà devait différer quelque peu de celle de la Grèce continentale. On y était beaucoup plus soucieux du repos des morts $\left({ }^{43}\right)$. Les imprécations se multiplièrent, surtout à l'époque impériale, à caractère magique ou plus légal, avec parfois menaces d'amendes à verser au fisc, au dèmos ou à la caisse d'un temple $\left.{ }^{(44}\right)$. On peut même envisager un gardiennage des tombes: une inscription de 120 ap. J.-C. signale vraisemblablement dans la région d’Alexandrie la présence d'un groupement de Lyciens chargé de la surveillance des tombeaux $\left({ }^{45}\right)$. Initiative municipale ou simplement privée si, comme le précise M. Ducos, la cité romaine

(34) F. Cumont, "ll sole vindice dei delitti ed il simbolo delle mani alzaten, Atti della Pontificia Accademia Romana di Archeologia, serie III, Memorie. I, I, Miscellanea G. Battista De Rossi, 1923, pp. 65-80 ; «Nuovi epitafi col simbolo della preghiera al dio vindice". Atti della Pontificia Accademia Romana di Archeologia. Rendiconti, V, 1927, pp. 69. 78 ; “Deux monuments ...", (voir note 15), pp, 381-395. Un article récent: B. Forsen, E. Sironen, «Zur Symbolik von dargestellten Hände", Arctos. XXIII. 1989, pp. 55-66 fait le point sur la signification des représentations de mains mais est centré surtout sur la main unique, ex-voto ou main bénéfique divine (main de Sabazios). Enfin on citera un recueil de conférences et d'extraits d'ouvrages des $x \mathrm{vi}^{\mathrm{E}}, \mathrm{xv}^{\mathrm{t}}$ et $\mathrm{xv} \|^{\mathrm{c}}$ siècles paru sous le titre La main, Orléans, 1996, Institut d'Arts visuels.

(35) K. Sıтt, Die Gebärden der Griechen und Römer. Leipzig, 1890.

(36) F. T. VAn STRATFN, "Gifts for the Gods", Faith. Hope and Worship. Aspects of Religious Mentality in the Ancient World, H. S. Verssfi éd., leyde, 1981, p. 83.

(37) F. Cumont, "Il sole vindice...", (voir note 34), pp. 70 et 76

(38) G. BıOrck, Der Fluch des Christen Sabinus, Papyrus Upsaliensis 8, Leipsig-Uppsala-Cambridge, 1938, pp. 27-34.

(39) F. CUMONT, «Il sole vindice...», pp. 65-80.

(40) ID., "Deux monuments...", (voir note 15), p. 385.

(41) 1D., ibid, p. 387 .

(42) G. HofFMANN, loc. cit., p. 317.

(43) J. H. M. Strubbe, "Cursed be he that moves my bones". Magika Hiera. Ancient Greek Magic and Religion. Chr. A. Faraone, D. Obsink éd., New York-Oxford, 1991, pp. 33-59.

(44) Ed. Frrzouts, M.-J. Morant, loc. cit., p. 249, note 46.

(45) A. Brannin, La prose sur pierre en Egypte à lëpoque hellènistique et romaine, Paris, 1992, n 61 : “...Ulpius Potâmon et ses collaborateurs, du groupement des Lyciens, ayant déclaré en présence de Dionysos ... que la surveillance des tombeaux, qui leur incombait de toute antiquité, avait été abusivement soustraite aux I.yciens par le secrétaire Dionysos ici présent...». 
n'étant chargée que de la destination sépulcrale du locus religiosus, la protection du tombeau ne relève que de dispositions privées $\left({ }^{46}\right)$.

Les mains supines levées dont la paume est visible et telles que nous pensons pouvoir définir les deux mains de la base de Kadyanda ne sauraient appartenir qu'à une personne placée face au monument, comme nous l'avons vu précédemment. C'est cette personne - nous pencherions pour le dédicant - qui en immortalisant un geste apporterait une protection ou du moins ajouterait une protection au monument funéraire. Les mains levées dont seul le dos est visible, avec les pouces à l'extérieur, sont aussi supines si ce sont les mains du défunt ou de toute autre représentation humaine ou divine que personnalise le monument. Les autres mains levées, sans trace de supination, avec paumes visibles et pouces à l'intérieur seraient celles du défunt appelant à la vengeance en cas de mort violente ou précoce, ou même, rien ne le contredit, tentant de protéger de tout vol ou déprédation le monument posé sur sa propre sépulture. On notera toutefois que la main n’a pas qu'un rôle bénéfique. Diverses épitaphes, très fréquentes en Phrygie et dont une à Athènes, dédiée à une défunte originaire d'Asie Mineure, tentent d'écarter la main violente du tombeau $\left({ }^{47}\right)$.

$\mathrm{Si}$ on reprend l'idée de P. Zanker (48), qui s'interroge sur les lieux où sont exposés les représentations figurées, sur le sens et l'importance qu'on leur attribue, que peut-on dire des mains levées? Au cours de l'Antiquité les protections de tombes ont pris des formes variées. Les mains levées, supines ou non, pourraient en avoir été une, même si leur représentation a recouvert aussi d'autres significations. Le souci des Lyciens de mettre leurs morts en sécurité s'est exprimé pendant un temps en creusant des tombes "pigeonniers", loculi nombreux dans les falaises à Tlos ou à Sidyma. Mais sur de tels tombeaux aucune épitaphe commémorant le nom du défunt ne pouvait être lue par les passants, aucun rituel entourant la pierre ne pouvait se dérouler. Les tombes se sont rapprochées des vivants avec tous les risques de vol et de déprédation que cela suppose. À la charge de chacun de protéger le tombeau qu'il avait érigé, explicitement en menaçant d'une amende, en faisant appel à la vengeance d'un dieu, ou implicitement en payant un gardiennage ou en faisant représenter deux mains. Ce pourrait être le cas pour cette petite base funéraire.

Marie-José MORANt (CNRS Strasbourg)

\section{Addendum}

Le manuscrit de cet article était déjà remis à l'imprimeur lorsque, grâce à Michel Matter. que je remercie cordialement, j’ai pris connaissance de l'article de S. Mittmann. L'auteur souligne, à partir d'exemples palestiniens et carthaginois, le caractère "multifonctionnel" de la main : unique, par paire - gauche et droite ou même deux droites - qui peut symboliser diverses choses : puissance divine. prière... «Das breit gefächterte Bedeutungspektrum, wie es uns, gewiß noch unvollständig, entgegentritt. liegt in der Natur der Sache, dem multifunktionalen Charakter der Hand, der sie als Symbol entsprechend breit verwendbar macht" ${ }^{49}$ ). On en conclura que l'interprétation de ce symbole doit prendre en compte des critères non seulement culturels et chronologiques mais aussi iconographiques et s'attacher à la destination (funéraire, honorifique) de l'objet sur lequel il figure.

(46) "Le tombeau. locus religiosus". La mort au quotidien dans le monde romain. Actes du colloque organise par I'Université de Paris IV. Paris-Sorhonne. 7-9 octohre 1993. F. Hinaris éd., Paris, 1995, p. 144.

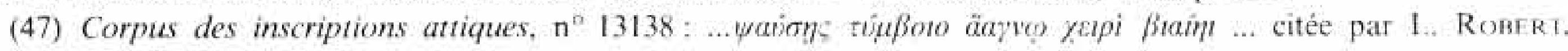
"Malédictions funéraires grecquesm. (RAI, 1978, pp. 243-255.

(48) «Nouvelles orientations de la recherche en iconographic. Commanditaires et spectateurs", RA, 1994, pp. 281293.

(49) S. Mirman, «Das Symbol der Hand in der altorientalischen Ikonographie". La Main de Dieu. Die Hand Gottes, R. Kinffr, J. Blikgiman éd., Wissenschaftliche Untersuchungen zum Neuen Testament, 94. Tübingen, 1997. pp. 19-47. 


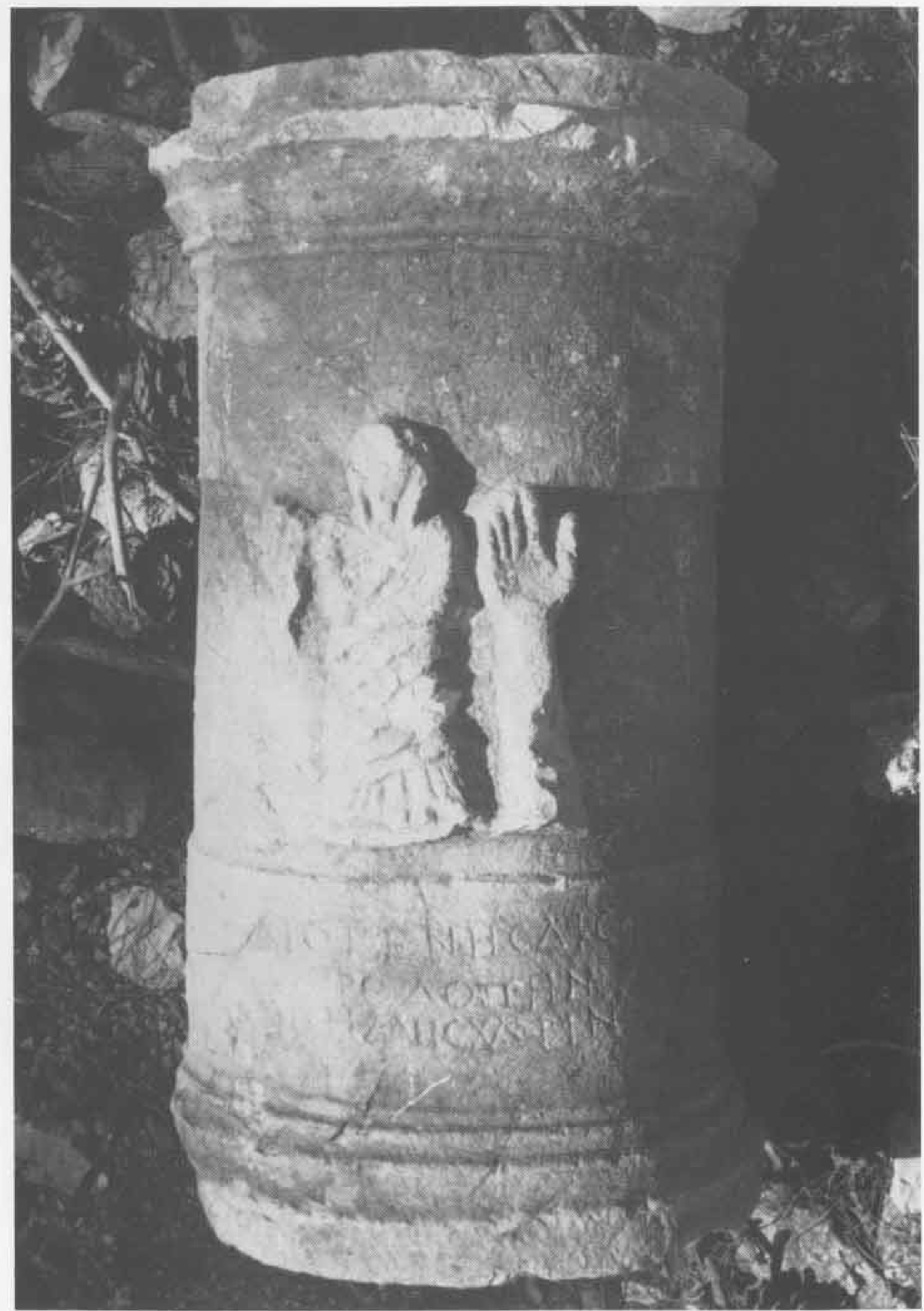

Fig. 1. - Kadyanda : base funéraire de Rhodopè (cliché Ed. Frezouts). 


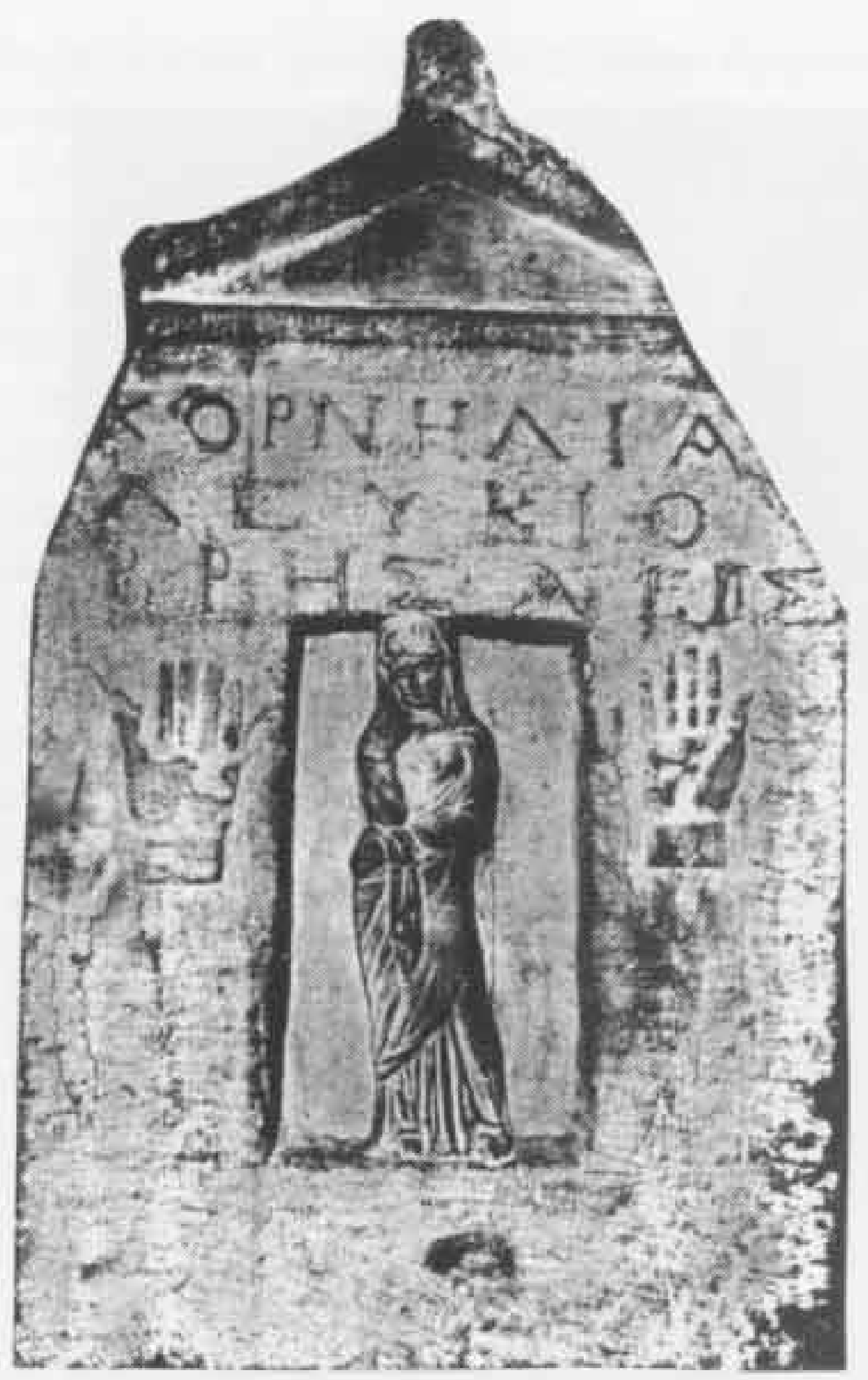

Fig. 2. - Orient grec? (d'après E. Prunt, H. Mовıus, Die ostgriechischen Grabreliefs, Mayence, 1977, $\mathrm{n}^{\circ} 433$ ).

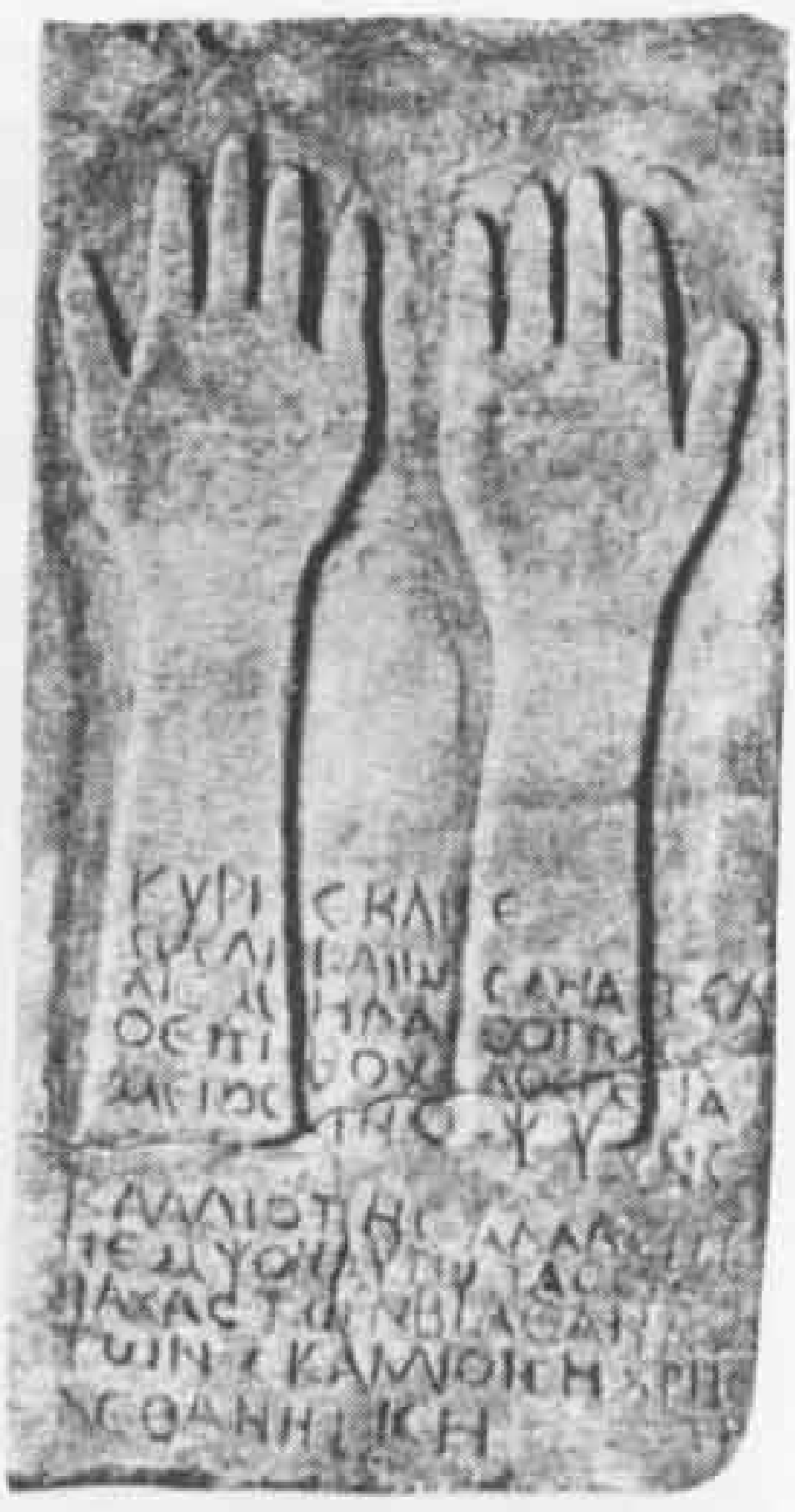

Fig. 3. - Enkomi (d'après F. CUMONT, Syria, XIV, 1933, p. 388).

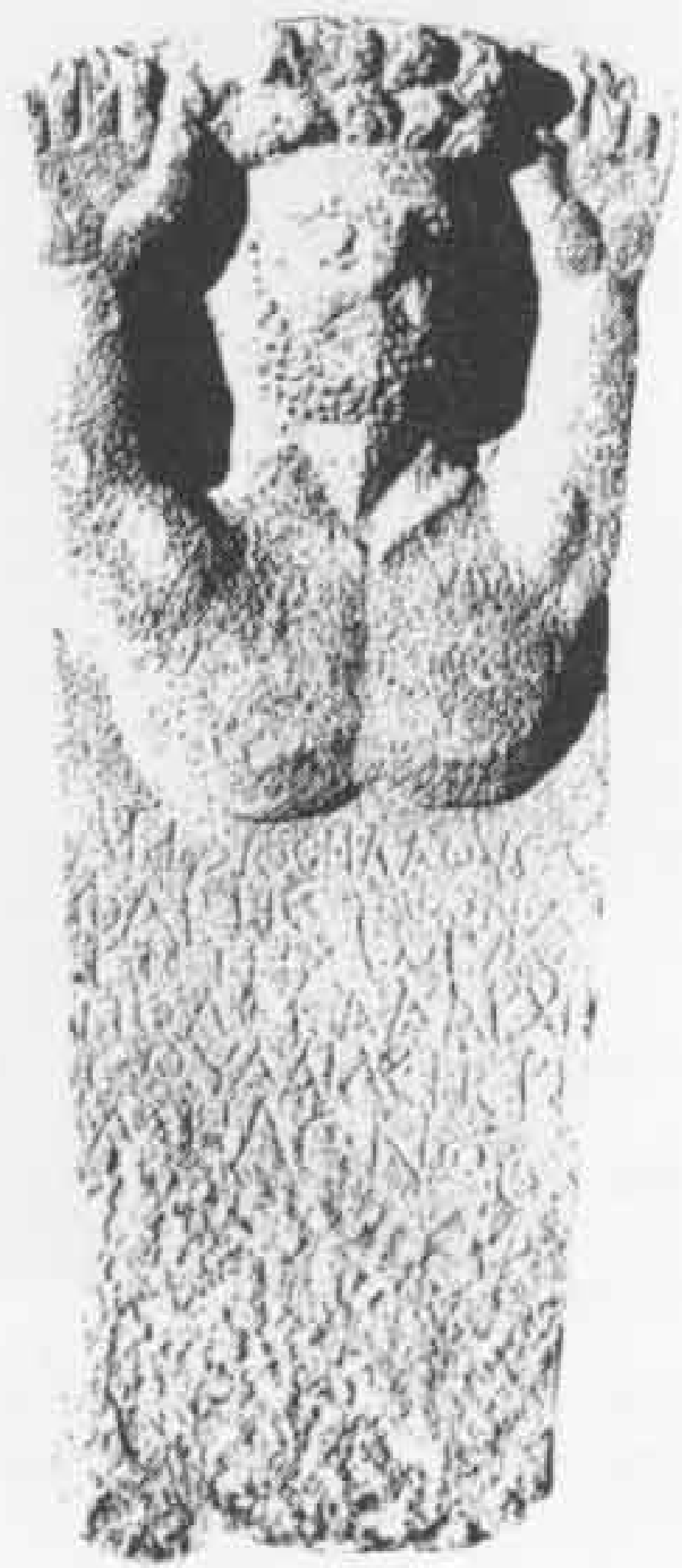

